\title{
Vólvulo de sigmoides
}

\author{
Sigmoid volvulus
}

\section{Correspondencia:}

Alejandra Bacilio-Cardozo.

Número de celular: 944149493

Correo Electrónico:

abc.medic@gmail.com

Domicilio: Calle Las Argentitas 150, Urb. La Rinconada. Trujillo, Perú.

\section{Contribuciones De Autoría:}

BCA: Contribuciones en la concepción y diseño del manuscrito, recolección, análisis e interpretación de los datos, redacción y revisión crítica del contenido del manuscrito y aprobación final del artículo.

Conflicto De Intereses: No declarados.

Financiamiento: Autofinanciado.

\section{Como Citar}

Bacilio-Cardozo Alejandra. Vólvulo de sigmoides. Rev méd panacea 2018; (3): 107-111

Recibido: 26 - 09 - 2018

Aceptado: 30 - 11 - 2018

Publicado: 28 - 12 - 2018

\section{RESUMEN}

Objetivo: Realizar la recopilación de la información más relevante descrita en diversos artículos científicos acerca del vólvulo de sigmoides, e integrarla para la puesta al día del cirujano. Materiales y métodos: Se realiza una estrategia de búsqueda para el desarrollo de la revisión, haciendo uso de PubMed y Cochrane Library. Resultados: La etiología del vólvulo de sigmoides es multifactorial, siendo el sigmoide redundante un factor predisponente. La incidencia más alta se da entre los 40 a 80 años. Es más común en hombres. En casi el $100 \%$ de pacientes, el dolor abdominal es el síntoma principal, asociado a distención abdominal y dificultad para eliminar flatos. La radiografía simple de abdomen es diagnóstica en el $57 \%$ - 90\% de pacientes. La detorsión endoscópica flexible es la principal opción de tratamiento, con tasas de éxito del 33\% al $91 \%$. La cirugía de emergencia se reserva para los casos complicados. Conclusiones: El vólvulo de sigmoides, es la rotación del sigmoide alrededor de sí mismo y su mesenterio. Los síntomas principales son dolor abdominal, distensión y dificultad para eliminar flatos. Los exámenes auxiliares incluyen la radiografía simple de abdomen, la tomografía y la resonancia. La endoscopia muestra un giro espiral de la mucosa en forma de esfínter. Se debe realizar unidad clínica, radiológica, endoscópica y, a veces, quirúrgica, para su diagnóstico. La devolvulación endoscópica se sugiere como principal opción terapeutica. La cirugía de emergencia se reserva para casos complicados. Esta patología aun presenta una elevada morbimortalidad.

\section{Palabras clave: Obstrucción intestinal, colon sigmoide, vólvulo}

\section{ABSTRACT}

Objective: To compile the most relevant information described in various scientific articles about the sigmoid volvulus, and integrate it for the surgeon's update. Materials and methods: A search strategy was developed for the development of the review, using PubMed and the Cochrane Library. Results: The etiology of the sigmoid volvulus is multifactorial, being the redundant sigmoid a predisposing factor. The highest incidence occurs between 40 to 80 years. It is more common in men. In almost $100 \%$ of patients, abdominal pain is the main symptom, associated with abdominal distension and difficulty in eliminating flatus. Plain radiography of the abdomen is diagnostic in $57 \%-90 \%$ of patients. Flexible endoscopic detortion is the main treatment option, with success rates of $33 \%$ to $91 \%$. Emergency surgery is reserved for complicated cases. Conclusions: The sigmoid volvulus is the rotation of the sigmoid around itself and its mesentery. The main symptoms are abdominal pain, distension and difficulty to eliminate flatus. Auxiliary exams include plain abdominal radiography, tomography and MRI. The endoscopy shows a spiral twist of the mucosa in the form of a sphincter. A clinical, radiological, endoscopic and, sometimes, surgical unit must be performed for its diagnosis. Endoscopic devolvulation is suggested as the main therapeutic option. Emergency surgery is reserved for complicated cases. This pathology still presents a high morbidity and mortality.

Keywords: intestinal obstruction, sigmoid colon, volvulus 


\section{INTRODUCCIÓN}

La obstrucción intestinal producida por el vólvulo de colon sigmoides, es una de las emergencias quirúrgicas más frecuentes en los hospitales andinos del Perú. Existen escritos que revelan que esta patología es conocida desde el antiguo Egipto, posteriormente descrita por los Hindúes. Desde épocas remotas, los quechuas la llamaban "CHITI WICSA" (vientre hinchado), y los Aymaras "CHITI USO" (mal de llenura), lo relacionaban con la ingesta de papas "nuevas" (ACHU CHOGUE), por su frecuente presentación en la época de cosecha.

El vólvulo de sigmoides es la rotación axial de un segmento del intestino lleno de aire sobre su mesenterio estrecho. Existen factores necesarios, predisponentes y precipitantes para la formación de un vólvulo. En el área Andina el vólvulo de sigmoides es más frecuente en hombres del área rural y mayores de 60 años, diferente a otras áreas endémicas como Turquía. La cirugía es uno de los pilares del manejo, se reserva generalmente en casos complicados. El diagnóstico temprano, y el grado de isquemia y necrosis intestinal tienen influencia directa en la morbilidad y la mortalidad, al igual que el tipo de procedimiento quirúrgico requerido, así como también algunas características epidemiológicas. Es justo por su alta morbimortalidad, por lo que se debe poner especial importancia en la revisión de esta patología.

\section{MATERIALES Y MÉTODOS}

Se realiza una estrategia de búsqueda para el desarrollo de la revisión de artículo, haciendo uso de PubMed y Cochrane Library.

\section{OBJETIVOS:}

\section{Objetivo General:}

Realizar la recopilación de la información esencial más relevante descrita en diversos artículos científicos disponibles acerca del vólvulo de sigmoides e integrarla para la puesta al día del cirujano en la patología.

\section{DEFINICIÓN}

El vólvulo del colon sigmoide es la rotación axial de un segmento del intestino lleno de aire sobre su mesenterio estrecho (1,2,3), el cual casi nunca ocurre cuando el colon está lleno con heces sólidas (2). Se produce cuando hay una elongación gradual y dilatación del colon sigmoide con la subsecuente torsión entorno al eje del mesenterio, desarrollando bridas entre los segmentos intestinales involucrados (4). El vólvulo del sigmoide generalmente produce obstrucción y oclusión vascular mesentérica temprana (1), comprometiendo el flujo sanguíneo del colon llevando a isquemia tisular, hipoxia y necrosis (5), con la subsecuente perforación y peritonitis (4).

\section{EPIDEMIOLOGÍA}

El vólvulo de sigmoides representa del $2 \%$ al $5 \%$ de las obstrucciones del colon en los países occidentales y del
$20 \%$ al $50 \%$ de las obstrucciones en los países del Este. Los países de África, Asia, Oriente Medio, América del Sur, Europa del Este y del Norte, así como Turquía, son regiones endémicas (6).

Generalmente afecta a adultos, con la incidencia más alta observada en las décadas de la $4^{a}$ a la $8^{a}$. Es más común en hombres y se presenta en proporciones que varían de 2/1 a 10/1 (6).

\section{ETIOLOGÍA}

Varios factores interactúan para la formación de un vólvulo del sigmoide, los cuales se pueden dividir en factores necesarios, factores predisponentes y factores precipitantes. El factor necesario es el colon sigmoide redundante con un mesocolon de base estrecha (2). Los principales factores predisponentes en nuestro medio son la mesocolonitis retráctil, y una entidad recientemente descrita para el área andina denominada el Dolicomegacolon Andino (DCMA) (7). La ingesta copiosa de alimentos fermentables, el uso excesivo de laxantes y catárticos, el embarazo y la presencia de masa pélvica, constituyen los principales factores precipitantes para la formación del vólvulo $(1,2,3,4,5)$

Algunos autores han mostrado correlaciones positivas entre la edad avanzada y la redundancia del colon sigmoide, así como la dolicomesentería, lo que puede explicar la relación entre la edad avanzada y esta patología. De manera similar, la dolicomesentería y la entrada pélvica más pequeña son más comunes en los varones, y estos causan torsión y no permiten la detorsión espontánea. Esto puede explicar su relación con el género masculino. Por otro lado, durante el embarazo se cree que el útero agrandado empuja al colon sigmoide redundante fuera de la pelvis y causa el vólvulo (6).

La altitud elevada puede conducir a una presión colónica alta, que causa un colon sigmoide redundante, y puede ser una posible razón para su dispersión geográfica (7). Del mismo modo, un hábito de dieta vegetal con alto contenido de fibra puede causar colon sigmoide redundante y puede explicar la relación con la dispersión geográfica y el estado socioeconómico $(6,7)$.

Algunas enfermedades, que incluyen adherencias postoperatorias, hernias internas, anomalías onfalo mesentéricas, malrotaciones, intususcepciones, megacolon congénito, apendicitis y carcinomas, pueden ser factores predisponentes raros $(1,3,8)$.

\section{FISIOPATOLOGÍA}

Dos problemas importantes surgen en el vólvulo de sigmoides: Obstrucción luminal y oclusión vascular $(1,9)$. Tanto la obstrucción mecánica como la fermentación bacteriana causan la distensión del bucle retorcido y el colon proximal. El aumento de la presión intracolónica disminuye la perfusión capilar. Tanto la oclusión mecánica como la trombosis de los vasos contribuyen a la isquemia. La lesión isquémica de la mucosa causa 
translocación bacteriana y toxemia, lo que resulta en gangrena colónica. El aumento de la presión intraabdominal causa el síndrome compartimental abdominal (6).

\section{CLASIFICACIÓN}

El vólvulo de sigmoide puede ser primario, cuando ocurren como resultado de una malrotación congénita del intestino o secundario, cuando ocurren por la rotación del colon sigmoide redundante entorno a su meso estrecho, por torsión del mismo alrededor de una banda o entorno a un estoma (1). También se puede clasificar en complicado y no complicado en cuanto a la presencia o no de signos de necrosis $(1,2)$.

En 2008, utilizando criterios preoperatorios y quirúrgicos que están correlacionados con la mortalidad, se desarrolló una clasificación para este, tratado quirúrgicamente, de la siguiente manera: Clase 1, pacientes sin factor de riesgo (edad avanzada, enfermedad asociada); Clase 2, aquellos sin shock o gangrena intestinal pero con otros factores de riesgo mencionados anteriormente; Clase 3, aquellos con shock; Clase 4, aquellos con gangrena intestinal; Clase 5, aquellos con shock y gangrena intestinal (10).

\section{PRESENTACIÓN CLÍNICA}

En casi el $100 \%$ de pacientes el dolor abdominal es el síntoma principal, asociado en frecuencia a la sensación de distención abdominal y dificultad para la eliminación de flatos Fig.1 (11).

Los pacientes generalmente se presentan con una demora media de 1 a 4 días. Las quejas adicionales incluyen vómitos, náuseas, diarrea, anorexia, hemorragia rectal y hematemesis $(1,3,11)$.

Los principales hallazgos físicos son distensión abdominal asimétrica y sensibilidad. Otros hallazgos incluyen sonidos intestinales anormales, timpanismo, recto vacío, peristalsis visible, masa abdominal y olor a heces de la respiración. La presencia de heces melanóticas rectales o sensibilidad al rebote y defensa muscular generalmente muestran gangrena 0 perforación y peritonitis (6).

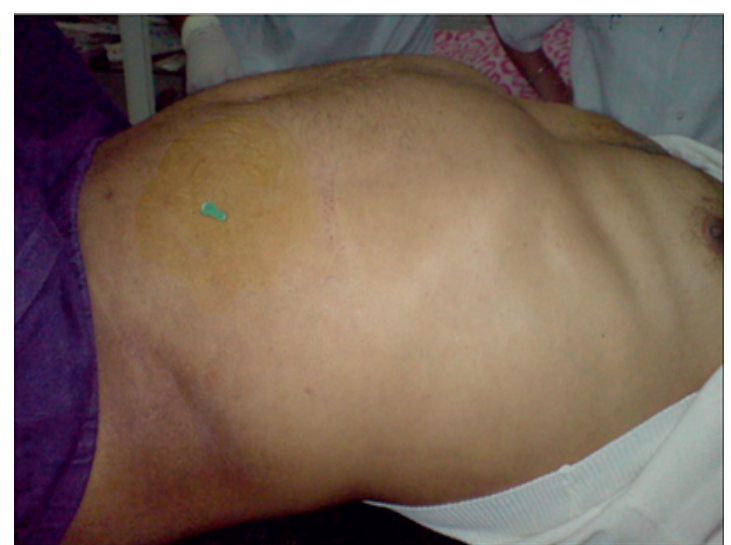

Figura 1. Distensión Abdominal por vólvulo de sigmoides.

\section{DIAGNÓSTICO}

Puede ser difícil hacer un diagnóstico preoperatorio preciso sin uso de sigmoidoscopia, CT O MRl; el diagnóstico incluso en algunos casos se realiza durante la laparotomía o en autopsias en $10-15 \%$ de pacientes (12).

Las radiografías simples de rayos $\mathrm{X}$ abdominales por lo general muestran un colon sigmoide dilatado y múltiples niveles de fluidos de aire intestinal pequeños o grandes (Figura 2). Los signos de rayos $\mathrm{X}$ de diagnóstico descritos son un signo de omega o herradura, signo de pico de pájaro, signo $\mathrm{V}$ invertido, signo $\mathrm{Y}$, signo de exposición norte, signo de café en grano, tubo interno doblado o as de signo de espadas, superposición pélvica izquierda o signo de superposición de flanco izquierdo, signo de superposición de hígado y signo de fosa ilíaca izquierda vacía. Se ha encontrado que la radiografía simple de abdomen es diagnóstica en el $57 \%$ - $90 \%$ de los pacientes (6).

La tomografía computada abdominal (TC) y la resonancia magnética (RM) generalmente muestran un mesenterio sigmoide giratorio además de asas sigmoideas dilatadas y niveles hidroaéreos intestinales pequeños o grandes. Tanto la TC como la RM tienen altos valores de diagnóstico Fig. $3(13,14)$.

La endoscopia generalmente muestra un giro en espiral de la mucosa en el colon sigmoide obstructivo, generalmente de $20 \mathrm{~cm}$ a $30 \mathrm{~cm}$ del borde anal. La endoscopia flexible tiene un alto valor diagnóstico $(12,14)$.

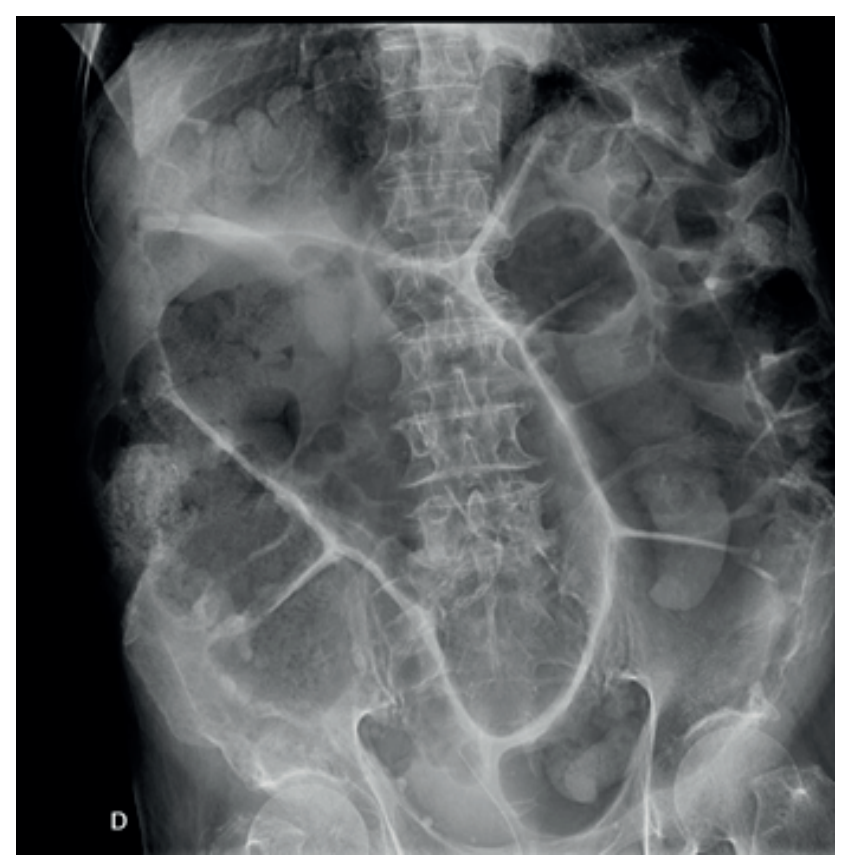

Figura 2. Radiografía Simple de Abdomen de pie mostrando imagen en "Grano de Café" 

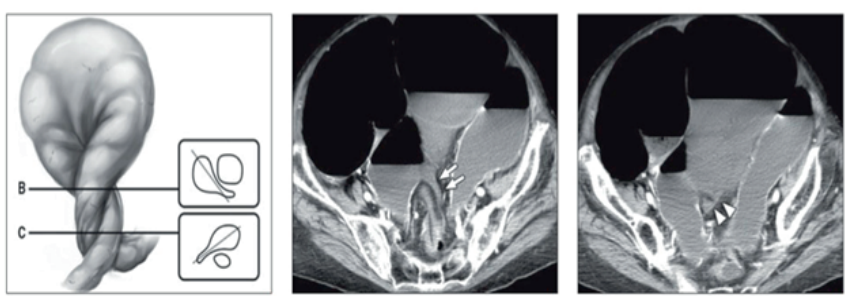

Figura 3. Tomografía mostrando asas intestinales gruesas torsionadas.

\section{TRATAMIENTO}

El vólvulo de sigmoides ocurre en pacientes ancianos y debilitados con una morbilidad, mortalidad e implicaciones de estilo de vida significativas. Debido a su tendencia a la hipovolemia y al shock tóxico, requieren una reanimación efectiva del medio interno (15).

Si no hay signos de isquemia intestinal y perforación, el enema con contraste hidrosoluble seguido de descompresión endoscópica y detorsión del vólvulo representa el paso inicial del tratamiento incluso en el entorno pediátrico. Sin embargo, el procedimiento requiere un alto grado de experiencia y está asociado a una alta tasa de recurrencia temprana incluso cuando se realiza con éxito. La cirugía electiva con resección sigmoidea, anastomosis primaria y sigmoidopexia es obligatoria (16).

El manejo quirúrgico de emergencia incluye técnicas no definitivas o definitivas como devolvulación, pexia del sigmoide, mesosigmoidoplastia o resección sigmoidea con anastomosis primaria o estoma, la resección con anastomosis primaria, que tiene una tasa de mortalidad del $8 \%$ al 33\%, es el procedimiento recomendado más comúnmente debido a la alta tasa de recurrencia de los procedimientos no definitivos. Otros procedimientos alternos son la sigmoidostomía con tubo, la extraperitonealización del colon sigmoide y la colostomía endoscópica percutánea (17).

\section{CONCLUSIONES}

El vólvulo de sigmoides, es la rotación del colon sigmoide alrededor de sí mismo y su mesenterio.

Esta patología se presenta con una interesante dispersión geográfica. Los adultos se ven afectados con frecuencia, sobretodo los varones.

Los síntomas principales son dolor abdominal, distensión y dificultad para eliminar flatos y para defecar; mientras que los signos principales son dolor $y$ distensión abdominal.

Los exámenes de ayuda diagnostica incluyen las radiografías simples de abdomen que muestran un colon sigmoide dilatado y niveles hidroaereos en el intestino, la tomografía y la resonancia magnética abdominal muestran un mesenterio sigmoide giratorio. La endoscopia muestra un giro espiral de la mucosa en forma de esfínter. Se debe realizar unidad clínica, radiológica, endoscópica y, a veces, quirúrgica, para llegar a su diagnóstico.

En el manejo, se recurre a la devolvulación endoscópica como la principal opción. La cirugía de emergencia se reserva para los casos que cursan con peritonitis, gangrena o perforación o para pacientes con fracaso de la endoscopia. La resección con anastomosis primaria es el procedimiento más comúnmente recomendado.

Esta patología aun presenta una elevada morbimortalidad. 


\section{REFERENCIAS BIBLIOGRÁFICAS}

1. Macutkiewicz Christian, Carlson gordon. "Acute abdomen: intestinal obstruction". Emergency surgery. 2008; vol 26, núm. 3, p. 102-107.

2. Schwartz seimur, et al. "Colon, recto y ano". Principios de Cirugía. 2000, ed. 7a; vol 2, cap. 26, p. 1360-1361.

3. Stephenson James, et al. "Intestinal obstruction". Surgery 2015; vol 29, p. 33-38.

4. Akcan alper, et al. "Feasibility of single-stage resection and primary anastomosis in patients with acute non complicated sigmoid volvulus". The american journal of surgery. 2007; vol 193, p. 421-426.

5. Chu Eric, et al. "Rapid colonoscopic detection and quantification of colonic ischemia by using a laser doppler flowmeter". Gastrointestinal endoscopy Journal 2007; vol 66, núm. 3, p. 630-632.

6. Selcuk Atamanalp. "Sigmoid volvulus review". The eurasian journal of medicine, 2010; vol 42: 142-7.

7. Frisancho Oscar. "Dolicomegacolon andino y vólvulos intestinales de altura". Revista de gastroenterología del Perú 2008; vol 28, p. 248-257.

8. Pahlman Lean, Enblad Peter, Rudberg Carl. Volvulus of the colon. Acta Quirúrgica Scandinavica 1989; 155: 53-6.

9. Arigbabu Alman, Badejo Oscar. Colonoscopy in the emergency treatment of colonic volvulus in nigeria. Disseases of the colon and rectum 1985; 28: 795-8.
10. Atamanalp Selcuk, Aydınlı Bulent, Ozturk Gurkan, et al. Classification of sigmoid volvulus. Turkish journal of medical sciences 2008; 38: 425-9.

11. Sáenz Lázaro, José. Diagnóstico clínico, epidemiológico y tratamiento del vólvulo de colon sigmoide en el Hospital Víctor Ramos Guardia, ministerio de salud huaraz. Tesis para optar el grado de bachiller en medicina, Trujillo, 2014; pg. 33-34.

12. Selcuk Atamanalp. The role of sigmoidoscopy in the diagnosis and treatment of sigmoid volvulus. Pakistan journal of medical sciences 2016; vol. 32 no. 1

13. Ozoguli biinyami. The role of $C T$ and $M R I$ in the diagnosis of sigmoid volvulus. Turkish journal of medical sciences 2014; 44(2):352.

14. Jaffe Tracy. Large-bowel obstruction in the adult: classic radiographic and CT findings, etiology, and mimics. Radiology 2015; vol 275: 3.

15. Dolejs Sara. Contemporary management of sigmoid volvulus. Journal of gastrointestinal surgery 2018; 22(8):1404-1411.

16. Parolini Filippo. Endoscopic management of sigmoid volvulus in children. World journal of gastrointestinal endoscopy. 2016; 8(12): 439-443.

17. Bruzzi M. Management of acute sigmoid volvulus: short- and long-term results. Colorectal disseases. 2015; 17(10):922-8.

\section{Las ediciones anteriores de revista médica PANACEA están disponibles en:}

\section{www.revpanacea.unica.edu.pe}

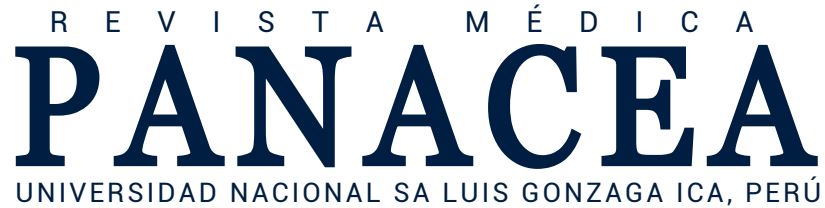

